\title{
Carroll limit of non-BPS Dp-brane
}

\section{J. Klusoň}

Department of Theoretical Physics and Astrophysics, Faculty of Science, Masaryk University, Kotlářská 2, 611 37, Brno, Czech Republic

E-mail: klu@physics.muni.cz

Abstract: We find Carroll non-BPS Dp-brane action by performing Carroll limit of a canonical form of unstable Dp-brane action. We analyze different Carroll limits and discuss solutions of the equations of motion of Carroll non-BPS Dp-brane at the tachyon vacuum.

KEYwords: D-branes, Tachyon Condensation

ArXiv ePrint: 1702.08685 


\section{Contents}

1 Introduction and summary 1

2 Carroll limit of non-BPS Dp-brane 3

2.1 Stringy Carroll limit 3

2.2 p-brane Carroll limit 5

3 Carroll limit of non-BPS Dp-brane with gauge field $\quad 6$

3.1 Modified stringy Carroll scaling limit 8

$\begin{array}{ll}3.2 & p \text {-brane Carroll limit } \\ & 12\end{array}$

\section{Introduction and summary}

The holographic ideas have very broad region of applications. For example, it was shown recently that holography is a very useful tool for the analysis of condensed matter systems, for recent review, see [1]. It turns out that non-relativistic systems play fundamental role in this analysis and hence they are now studied very intensively since they are also related to famous P. Hořava's proposal [2], for recent review, see [3]. ${ }^{1}$

On the other hand asymptotic symmetries of flat space-time were also studied very intensively especially in the context of holography in the flat space-time $[10-14]^{2}$ and it was shown that BMS symmetry $[16,17]$ has significant meaning in this analysis. Moreover, remarkable connection between BMS group and Carroll group was recently discovered in $[18,19]$. Carroll symmetry corresponds to the limit of Poincare algebra when the velocity of light goes to zero [20]. There is also very interesting relation between the non-relativistic symmetry and Carroll symmetry [21].

As in case of non-relativistic symmetry [22-24, 26-31], Carroll symmetry can be imposed at the level of action for relativistic particle $[32,33]$ where it was shown that the particle possesses trivial dynamics. This analysis was recently extended to another extended objects in string theory, namely fundamental string and $p$-branes in [35]. Explicitly, Carroll string and Carroll p-brane were constructed here by taking the Carrollian limits of their canonical actions. It was also argued here that the resulting object exhibit trivial dynamics irrespective whether we take different Carroll limits on their actions.

Despite the fact that the dynamics of Carroll particle, strings and p-branes is trivial it is instructive to apply Carrollian limits of canonical actions of other objects in string theory, as for example non-BPS Dp-branes [36-38] which is the goal of present paper. As the first step we consider different Carroll limits in case of unstable p-brane without gauge fields. We analyze the equations of motion that follow from these Carroll unstable

\footnotetext{
${ }^{1}$ See, for example [4-9, 34].

${ }^{2}$ For extensive list of references, see for example recent work [15].
} 
p-branes and their tachyon vacuum solutions. It is expected that at the tachyon vacuum with zero electric flux an unstable Dp-brane disappears and we are left with the gas of massless non-interacting particles $[40,41,43]$ that move in the whole target space-time. We show that this occurs in case of Carroll unstable p-brane as well with one important exception that we have to restrict to the configuration with zero canonical momenta of the Carroll particle along spatial directions where the Carroll contraction has been performed. Of course, the dynamics in these directions is again trivial since the time derivative of embedding coordinates does not depend on conjugate momenta.

In order to find Carroll non-BPS Dp-brane whose tachyon vacuum state is equivalent to the gas of fundamental string we have to consider Carroll limit of non-BPS Dp-brane action with world-volume gauge field included. It turns out that the resulting form of the Carroll non-BPS Dp-brane depends on the fact whether we scale gauge field in the same way as embedding coordinates or not. Explicitly, we firstly consider the case where the gauge field are not scaled. Then we found that even in the stringy Carroll limit the Carroll nonBPS Dp-brane at the tachyon vacuum does not have solutions that could be interpreted as fundamental string solutions. For that reason we consider the second possibility where the gauge field scales as well. Now we find that for the stringy Carroll scaling limit non-BPS Dpbrane at the tachyon vacuum possesses fundamental string solutions that can be interpreted as the Carroll string with agreement with the general ideas of the tachyon condensation. On the other hand when we consider p-brane Carroll scaling limit on non-BPS Dp-brane we find that this unstable Dp-brane has solution of its tachyon vacuum equations of motion that can be interpreted as Carroll fundamental string that has vanishing momenta along directions where the Carroll limit was taken and also embedding coordinates along these directions do not depend on spatial coordinate. Of course, all these solutions are in some sense equivalent since the time evolution of these embedding fields do not depend on conjugate momenta. On the other hand it is an interesting issue what it is the physical meaning of the solutions of the tachyon equation of motion with non-zero momenta along spatial directions where the Carroll contraction has been performed.

Let us outline our results. We analyzed different Carroll limits on the world-volume of unstable Dp-brane either with zero or non-zero gauge fields. We discuss the tachyon vacuum solutions and we argued that they correspond to the Carroll particle (in case of zero electric flux) or Carroll string (in case of non-zero electric flux) on condition that the momenta along spatial directions where the Carroll limit was performed are equal to zero. It is interesting to compare this result with the fate of the unstable Dp-brane at the tachyon vacuum moving in general space-time, that correspond to the gas of fundamental strings propagating in the whole target space-time.

It is possible to extend this work in severe directions. For example, since there is a strong similarity between Carroll limit and non-relativistic limit we would like to perform non-relativistic limit in case of an unstable Dp-brane, following recent interesting paper [28]. This work is currently in progress.

This paper is organized as follows. In the next section 2 we analyze Carroll limit on the world-volume of unstable Dp-brane. Then in section 3 we extend this analysis to the case of unstable Dp-brane with non-zero gauge field. 


\section{Carroll limit of non-BPS Dp-brane}

\subsection{Stringy Carroll limit}

In this section we consider stringy Carroll limit of non-BPS Dp-brane action without gauge fields. Let us start with an action for unstable Dp-brane ${ }^{3}$

$$
S=-\tilde{\tau}_{p} \int d^{p+1} \xi V(\tilde{T}) \sqrt{-\operatorname{det} \mathbf{A}}, \quad \mathbf{A}_{\alpha \beta}=g_{M N} \partial_{\alpha} \tilde{x}^{M} \partial_{\beta} \tilde{x}^{N}+\partial_{\alpha} \tilde{T} \partial_{\beta} \tilde{T},
$$

where $\tilde{\tau}_{p}$ is a non-BPS Dp-brane tension, $\tilde{x}^{M}, M=0, \ldots, D-1$ label position of non-BPS Dp-brane in the target space time with the metric $g_{M N}$. Further, $\xi^{\alpha}, \alpha=0, \ldots, p$ are world-volume coordinates on non-BPS Dp-brane. Finally $T$ is the tachyon field and $V(T)$ is its potential when we presume that $V$ is even function with three extremes, where $T_{0}=0$ is unstable maximum with $V\left(T_{0}\right)=0$ while $\pm T_{\min }$ are global minima of the potential with $V\left( \pm T_{\min }\right)=0$ [36-38] for review and extensive list of references, see [39].

In order to formulate Carroll limit we have to proceed to the Hamiltonian formalism of the action (2.1). From (2.1) we find conjugate momenta

$$
\begin{aligned}
\tilde{p}_{M} & =-\tilde{\tau}_{p} g_{M N} \partial_{\alpha} \tilde{x}^{N}\left(\mathbf{A}^{-1}\right)^{\alpha 0} \sqrt{-\operatorname{det} \mathbf{A}} \\
\tilde{p}_{T} & =-\tilde{\tau}_{p} \partial_{\alpha} T\left(\mathbf{A}^{-1}\right)^{\alpha 0} \sqrt{-\operatorname{det} \mathbf{A}} .
\end{aligned}
$$

Using these relations it is easy to see that the bare Hamiltonian is equal to zero

$$
H_{B}=\int d^{p} \xi\left(\tilde{p}_{M} \partial_{0} \tilde{x}^{M}+\tilde{p}_{T} \partial_{0} \tilde{T}-\mathcal{L}\right)=0
$$

while we have following set of primary constraints

$$
\mathcal{H}_{\tau}=\tilde{p}_{M} g^{M N} \tilde{p}_{N}+\tilde{\tau}_{p}^{2} V^{2} \operatorname{det} \mathbf{A}_{i j} \approx 0, \quad i, j=1, \ldots, p, \quad \mathcal{H}_{i}=\tilde{p}_{M} \partial_{i} \tilde{x}^{M} \approx 0 .
$$

Using these constraints we can write non-BPS Dp-brane canonical action in the form

$$
S=\int d^{p+1} \xi\left(\tilde{p}_{M} \partial_{0} \tilde{x}^{M}+\tilde{p}_{T} \partial_{0} \tilde{T}-\tilde{N} \mathcal{H}_{\tau}-\tilde{N}^{i} \mathcal{H}_{i}\right)
$$

where $\mathcal{H}_{\tau}, \mathcal{H}_{i}$ are given in (2.4) and where $\tilde{N}, \tilde{N}^{i}$ are corresponding Lagrange multipliers.

Using (2.5) we find Carroll limit of non-BPS Dp-brane action. Following [35] we introduce "stringy" Carroll limit defined as:

$$
\begin{aligned}
& \tilde{x}^{\mu}=\frac{X^{\mu}}{\omega}, \quad \tilde{p}_{\mu}=\omega P_{\mu}, \quad \mu, \nu=0,1, \\
& \tilde{x}^{I}=X^{I}, \quad \tilde{p}_{I}=P_{I}, \quad I=2, \ldots, D-1, \\
& \tilde{T}=T, \quad \tilde{p}_{T}=p_{T}, \\
& \tilde{N}=\frac{1}{\omega^{2}} N, \quad \tilde{N}^{i}=N^{i}, \quad \tilde{\tau}_{p}=\omega \tau_{p}
\end{aligned}
$$

\footnotetext{
${ }^{3}$ We work in units $2 \pi \alpha^{\prime}=1$.
} 
and take the limit $\omega \rightarrow \infty$. We also consider string in flat space-time so that $g_{M N}=\eta_{M N}$. As a result of this limiting procedure we obtain Carroll non-BPS Dp-brane action

$$
\begin{aligned}
S=\int d^{p+1} \xi & \left(P_{\mu} \partial_{0} X^{\mu}+P_{I} \partial_{0} X^{I}+P_{T} \partial_{0} T\right. \\
& \left.-N\left(P_{\mu} \eta^{\mu \nu} P_{\nu}+\tau_{p}^{2} V^{2} \operatorname{det} \mathbf{a}_{i j}\right)-N^{i}\left(P_{\mu} \partial_{i} X^{\mu}+P_{I} \partial_{i} X^{I}+P_{T} \partial_{i} T\right)\right),
\end{aligned}
$$

where

$$
\mathbf{a}_{i j}=\partial_{i} X^{I} \partial_{j} X_{I}+\partial_{i} T \partial_{j} T, \quad i, j=1, \ldots, p .
$$

The equations of motion that we derive from (2.7) have the form

$$
\begin{aligned}
\partial_{0} X^{\mu}-2 N \eta^{\mu \nu} P_{\nu}-N^{i} \partial_{i} X^{I} & =0, & -\partial_{0} P_{\mu}+\partial_{i}\left(N^{i} P_{\mu}\right) & =0, \\
\partial_{0} X^{I}-N^{i} \partial_{i} X^{I} & =0, & -\partial_{0} P_{I}+2 \tau_{p}^{2} \partial_{i}\left(N V^{2} \partial_{j} X^{I}\left(\mathbf{a}^{-1}\right)^{i j}\right)+\partial_{i}\left(N^{i} P_{I}\right) & =0, \\
\partial_{0} T-N^{i} \partial_{i} T & =0, & -\partial_{0} P_{T}-2 N \tau_{p}^{2} V \frac{d V}{d T} \operatorname{det} \mathbf{a}_{i j}+\partial_{i}\left(N^{i} p_{T}\right) & =0, \\
P_{\mu} \eta^{\mu \nu} P_{\nu}+\tau_{p}^{2} V^{2} \operatorname{det} \mathbf{a}_{i j} & =0, & P_{\mu} \partial_{i} X^{\mu}+P_{I} \partial_{i} X^{I}+P_{T} \partial_{i} T & =0 .
\end{aligned}
$$

We are interested in the interpretation of the equations of motion when Carroll non-BPS Dp-brane is in its tachyon vacuum state defined as $T=T_{\min }, \frac{d V}{d T}\left(T_{\min }\right)=0, V\left(T_{\min }\right)=0$ together with $P_{T}=0$. In this case the equations of motion (2.9) take the form

$$
\begin{aligned}
\partial_{0} X^{0}+2 N P_{0}-N^{i} \partial_{i} X^{0} & =0, & \partial_{0} X^{1}-2 N P_{1}-N^{i} \partial_{i} X^{1} & =0, \\
-\partial_{0} P_{0}+\partial_{i}\left(N^{i} P_{0}\right) & =0, & -\partial_{0} P_{1}+\partial_{i}\left(N^{i} P_{1}\right) & =0, \\
\partial_{0} X^{I}-N^{i} \partial_{i} X^{I} & =0, & -\partial_{0} P_{I}+\partial_{i}\left(N^{i} P_{I}\right) & =0, \\
-P_{0}^{2}+P_{1}^{2} & =0, & P_{\mu} \partial_{i} X^{\mu}+P_{I} \partial_{i} X^{I} & =0 .
\end{aligned}
$$

It is well known that the non-BPS Dp-brane at its tachyon vacuum with zero electric flux is equivalent to the gas of massless particles that propagate in the whole target spacetime [42]. Now we would like to see whether this interpretation holds in case of the Carroll non-BPS Dp-brane at the tachyon vacuum. In other words we would like to see whether these equations of motion have natural interpretation as the equations of motion of Carroll massless particle [32]. To see this in more details we determine corresponding action starting with an action for massless relativistic particle

$$
S=\int d \tau\left[\tilde{K}_{M} \partial_{\tau} \tilde{Z}^{M}-\lambda_{\tau} \tilde{K}_{M} \eta^{M N} \tilde{K}_{N}\right]
$$

Following [32] we introduce particle Carroll limit as

$$
\begin{aligned}
& Z^{0}=\frac{t}{\omega}, \quad \tilde{K}_{0}=-\omega E, \quad \lambda_{\tau}=\frac{e}{\omega^{2}}, \\
& \tilde{K}_{I}=K_{I}, \quad \tilde{Z}^{I}=Z^{I}, \quad I=1, \ldots, D-1 .
\end{aligned}
$$

In this limit the action (2.11) has the form

$$
S=\int d \tau\left(-\partial_{\tau} t E+K_{I} \partial_{\tau} Z^{I}+e E^{2}\right)
$$


with corresponding equations of motion

$$
\begin{array}{rlrl}
-\partial_{\tau} t+2 e E & =0, & \partial_{\tau} E=0, \\
\partial_{\tau} Z^{I} & =0, & \partial_{\tau} K_{I}=0, \\
E^{2} & =0 . & &
\end{array}
$$

First of all, comparing (2.10) with the last equation in (2.14) we immediately see that in order the solutions of the equations of motion (2.10) have massless particle interpretation we have to demand that $P_{1}=0$. Then following [40,42] we now propose an ansatz for the solution of the equation of motion (2.10)

$$
\begin{aligned}
& \left.P_{0}\left(\xi^{0}\right)\right|_{\xi^{0}=\tau}=-E(\tau),\left.\quad X^{0}\left(\xi^{0}\right)\right|_{\xi^{0}=\tau}=t(\tau), \\
& \left.X^{1}\left(\xi^{0}\right)\right|_{\xi^{0}=\tau}=Z^{1}(\tau) \text {, } \\
& \left.X^{I}\left(\xi^{0}\right)\right|_{\xi^{0}=\tau}=Z^{I}(\tau),\left.\quad P_{I}\left(\xi^{0}\right)\right|_{\xi^{0}=\tau}=K_{I}(\tau), \quad I=2, \ldots, D-1, \\
& \left.N\left(\xi^{0}\right)\right|_{\xi^{0}=\tau}=e(\tau), \quad N^{i}=0, \quad i=1, \ldots, p .
\end{aligned}
$$

We see that this is solution of the equations of motion (2.10) on condition that $Z^{I}, Z^{0}$, $K_{0}, Z^{i}$ obey the equation of motion (2.14) with condition $P_{i}=0$.

\section{2 p-brane Carroll limit}

In previous section we introduced Carroll limit à la string when we scale first two target space coordinates and conjugate momenta. We can also define Carroll limit à la particle when we only scale time coordinate [35]. In this section we consider Carroll limit à la p-brane that is defined as [35]

$$
\begin{aligned}
& \tilde{x}^{\mu}=\frac{X^{\mu}}{\omega}, \quad p_{\mu}=\omega P_{\mu}, \quad \mu=0, \ldots, p, \\
& \tilde{x}^{I}=X^{I}, \quad p_{I}=P_{I}, \quad I=p+1, \ldots, D-1, \\
& \tilde{N}=\frac{1}{\omega^{2}} N, \quad \tilde{N}^{i}=N^{i}, \quad \tilde{\tau}_{p}=\omega \tau_{p}, \\
& \tilde{T}=T, \quad p_{T}=P_{T} .
\end{aligned}
$$

The difference between stringy and p-brane Carroll limit is in the number of scalar modes that are scaled. Clearly we could define $q$-brane Carroll limit for any $0 \leq q \leq p$ where $q=0$ corresponds to Carroll particle limit, $q=1$ corresponds to stringy Carroll limit and so on. Performing the scaling limit (2.16) in the action (2.5) we obtain p-brane Carroll non-BPS Dp-brane action in the form

$$
\begin{aligned}
S=\int d^{p+1} \xi & \left(P_{\mu} \partial_{0} X^{\mu}+P_{I} \partial_{0} X^{I}+P_{T} \partial_{0} T\right. \\
& \left.-N\left(P_{\mu} \eta^{\mu \nu} P_{\nu}+\tau_{p}^{2} V^{2} \operatorname{det} \mathbf{a}_{i j}\right)-N^{i}\left(P_{\mu} \partial_{i} X^{\mu}+P_{I} \partial_{i} X^{I}+P_{T} \partial_{i} T\right)\right),
\end{aligned}
$$

where

$$
\mathbf{a}_{i j}=\partial_{i} X^{I} \partial_{j} X_{I}+\partial_{i} T \partial_{j} T, \quad i, j=1, \ldots, p, \quad I, J=p+1, \ldots, D-1 .
$$


The equations of motion that we derive from (2.17) at the tachyon vacuum have the form

$$
\begin{aligned}
\partial_{0} X^{\mu}-2 N \eta^{\mu \nu} P_{\nu}-N^{i} \partial_{i} X^{I} & =0, & -\partial_{0} P_{\mu}+\partial_{i}\left(N^{i} P_{\mu}\right) & =0, \\
\partial_{0} X^{I}-N^{i} \partial_{i} X^{I} & =0, & -\partial_{0} P_{I}+\partial_{i}\left(N^{i} P_{I}\right) & =0, \\
P_{\mu} \eta^{\mu \nu} P_{\nu} & =0, & P_{\mu} \partial_{i} X^{\mu}+P_{I} \partial_{i} X^{I}+P_{T} \partial_{i} T & =0 .
\end{aligned}
$$

We can again presume that at the tachyon vacuum non-BPS Dp-brane disappears and we should find collection of Carroll particles. From the first equation on the last line above we find that this is possible when $P_{m}=0, m=1, \ldots, p$.

In order to support this arguments let us consider following ansatz

$$
\begin{aligned}
\left.P_{0}\left(\xi^{0}\right)\right|_{\xi^{0}=\tau} & =-E(\tau), & \left.X^{0}\left(\xi^{0}\right)\right|_{\xi^{0}=\tau} & =t(\tau), \\
\left.X^{m}\left(\xi^{0}\right)\right|_{\xi^{0}=\tau} & =Z^{m}(\tau), & m & =1, \ldots, p, \\
\left.X^{I}\left(\xi^{0}\right)\right|_{\xi^{0}=\tau} & =Z^{I}(\tau), & \left.P_{I}\left(\xi^{0}\right)\right|_{\xi^{0}=\tau} & =K_{I}(\tau), \\
\left.N\left(\xi^{0}\right)\right|_{\xi^{0}=\tau} & =e(\tau), & N^{i} & =0 .
\end{aligned}
$$

It is easy to see that (2.20) solve the equations of motion (2.19) on condition that $Z^{I}, Z^{0}$, $Z^{m}, K_{m}=0$ obey the equations of motion (2.14). In other words, the solutions of the equation of motion of Carroll non-BPS Dp-brane at the tachyon vacuum describes massless Carroll particles with vanishing momenta $K_{m}=0$. Note that they are the momenta along spatial directions where the Carroll limit has been performed.

\section{Carroll limit of non-BPS Dp-brane with gauge field}

We would like to see whether it is possible to have Carroll limit for non-BPS Dp-brane that at the tachyon vacuum has solution of the equations of motion corresponding to the fundamental string. As we know from the analysis of unstable Dp-brane the electric field on the world-volume of non-BPS Dp-brane is crucial [40, 42]. For that reason we have to consider Carroll limit for non-BPS Dp-brane with the gauge field. Recall that such an action has the form

$$
\begin{aligned}
S & =-\tilde{\tau}_{p} \int d^{p+1} \xi V(\tilde{T}) \sqrt{-\operatorname{det} \mathbf{A}_{\alpha \beta}} \\
\mathbf{A}_{\alpha \beta} & =g_{M N} \partial_{\alpha} \tilde{x}^{M} \partial_{\beta} \tilde{x}^{N}+\tilde{F}_{\alpha \beta}+\partial_{\alpha} \tilde{T} \partial_{\beta} \tilde{T}, \quad \tilde{F}_{\alpha \beta}=\partial_{\alpha} \tilde{A}_{\beta}-\partial_{\beta} \tilde{A}_{\alpha} .
\end{aligned}
$$

In order to define Carroll limit we have to find canonical form of the action which means that we have to find corresponding Hamiltonian. Explicitly, from (3.1) we find

$$
\begin{aligned}
\tilde{p}_{M} & =-\tilde{\tau}_{p} V g_{M N} \partial_{\beta} \tilde{x}^{N}\left(\mathbf{A}^{-1}\right)_{S}^{\beta 0} \sqrt{-\operatorname{det} \mathbf{A}}, \\
\tilde{p}_{T} & =-\tilde{\tau}_{p} V \partial_{\beta} \tilde{T}\left(\mathbf{A}^{-1}\right)_{S}^{\beta 0} \sqrt{-\operatorname{det} \mathbf{A}}, \\
\tilde{\pi}^{i} & =-\tilde{\tau}_{p} V\left(\mathbf{A}^{-1}\right)_{A}^{i 0} \sqrt{-\operatorname{det} \mathbf{A}}, \quad \tilde{\pi}^{0} \approx 0,
\end{aligned}
$$

where

$$
\left(\mathbf{A}^{-1}\right)_{S}^{\alpha \beta}=\frac{1}{2}\left(\left(\mathbf{A}^{-1}\right)^{\alpha \beta}+\left(\mathbf{A}^{-1}\right)^{\beta \alpha}\right), \quad\left(\mathbf{A}^{-1}\right)_{A}^{\alpha \beta}=\frac{1}{2}\left(\left(\mathbf{A}^{-1}\right)^{\alpha \beta}-\left(\mathbf{A}^{-1}\right)^{\beta \alpha}\right) .
$$


Using (3.2) we easily find that the bare Hamiltonian is equal to

$$
H_{B}=\int d^{p} \xi \pi^{i} \partial_{i} A_{0}
$$

while we have $p+1$ primary constraints

$$
\begin{aligned}
\mathcal{H}_{\tau} & =\tilde{p}_{M} g^{M N} \tilde{p}_{N}+\tilde{p}_{T}^{2}+\tilde{\pi}^{i} \mathbf{A}_{i j}^{S} \tilde{\pi}^{j}+\tilde{\tau}_{p}^{2} V^{2} \operatorname{det} \mathbf{A}_{i j} \approx 0, \\
\mathcal{H}_{i} & =\tilde{p}_{M} \partial_{i} \tilde{x}^{M}+\tilde{p}_{T} \partial_{i} \tilde{T}+\tilde{F}_{i j} \tilde{\pi}^{j}
\end{aligned}
$$

so that the canonical action is equal to

$$
S=\int d^{p+1} \xi\left(\tilde{p}_{M} \partial_{0} \tilde{x}^{M}+\tilde{p}_{T} \partial_{0} \tilde{T}+\tilde{\pi}^{i} \partial_{0} \tilde{A}_{i}-\tilde{N} \tilde{\mathcal{H}}_{\tau}-\tilde{N}^{i} \mathcal{H}_{i}-\tilde{\pi}^{i} \partial_{i} \tilde{A}_{0}\right) .
$$

As in the first section we firstly consider "stringy" Carroll limit

$$
\begin{aligned}
& \tilde{x}^{\mu}=\frac{X^{\mu}}{\omega}, \quad \tilde{p}_{\mu}=\omega P_{\mu}, \\
& \tilde{x}^{I}=X^{I}, \quad \tilde{p}_{I}=P_{I}, \quad I=2, \ldots, D-1, \\
& \tilde{T}=T, \quad \tilde{p}_{T}=p_{T}, \\
& \tilde{N}=\frac{1}{\omega^{2}} N, \quad \tilde{N}^{i}=N^{i}, \quad \tilde{\tau}_{p}=\omega \tau_{p}, \\
& \tilde{A}_{i}=A_{i}, \quad \tilde{\pi}^{i}=\pi^{i} .
\end{aligned}
$$

Then in the limit $\omega \rightarrow \infty$ we obtain action for "stringy" Carroll non-BPS Dp-brane in the form $\omega \rightarrow \infty$

$$
\begin{aligned}
S=\int d^{p+1} \xi & \left(P_{\mu} \partial_{0} X^{\mu}+P_{I} \partial_{0} X^{I}+p_{T} \partial_{0} T+\pi^{i} \partial_{0} A_{i}\right. \\
& \left.-N\left(P_{\mu} \eta^{\mu \nu} P_{\nu}+\tau_{p}^{2} V^{2} \operatorname{det} \mathbf{a}_{i j}\right)-N^{i}\left(P_{\mu} \partial_{i} X^{\mu}+P_{I} \partial_{i} X^{I}+p_{T} \partial_{i} T+F_{i j} \pi^{j}\right)-\pi^{i} \partial_{i} A_{0}\right),
\end{aligned}
$$

where

$$
\mathbf{a}_{i j}=\partial_{i} X^{I} \partial_{j} X_{I}+\partial_{i} T \partial_{j} T+F_{i j} .
$$

From (3.8) we determine following equations of motion

$$
\begin{aligned}
& \partial_{0} X^{\mu}-2 N \eta^{\mu \nu} P_{\nu}-N^{i} \partial_{i} X^{\mu}=0 \text {, } \\
& -\partial_{0} P_{\mu}+\partial_{i}\left(N^{i} P_{\mu}\right)=0, \\
& \partial_{0} X^{I}-N^{i} \partial_{i} X^{I}=0, \quad-\partial_{0} P_{I}+2 \tau_{p}^{2} \partial_{i}\left[N V^{2} \delta_{I J} \partial_{j} X^{J}\left(\mathbf{a}^{-1}\right)_{S}^{j i} \operatorname{det} \mathbf{a}\right]+\partial_{i}\left(N^{i} P_{I}\right)=0, \\
& \partial_{0} T-N^{i} \partial_{i} T=0, \quad-\partial_{0} p_{T}-2 N \tau_{p}^{2} V \frac{d V}{d T} \operatorname{det} \mathbf{a}_{i j}+\partial_{i}\left(N^{i} p_{T}\right)=0, \\
& \partial_{0} A_{i}-N^{j} F_{j i}-\partial_{i} A_{0}=0, \quad-\partial_{0} \pi^{i}+\partial_{j}\left(N^{j} \pi^{i}\right)-\partial_{j}\left(N^{i} \pi^{j}\right)=0, \quad \partial_{i} \pi^{i}=0, \\
& P_{\mu} \eta^{\mu \nu} P_{\nu}+\tau_{p}^{2} V^{2} \operatorname{det} \mathbf{a}_{i j}=0, \quad P_{\mu} \partial_{i} X^{\mu}+P_{I} \partial_{i} X^{I}+p_{T} \partial_{i} T+F_{i j} \pi^{j}=0 .
\end{aligned}
$$

These equations of motion simplify considerably at the tachyon vacuum

$$
\begin{array}{rlrl}
\partial_{0} X^{\mu}-2 N \eta^{\mu \nu} P_{\nu}-N^{i} \partial_{i} X^{\mu} & =0, & -\partial_{0} P_{\mu}+\partial_{i}\left(N^{i} P_{\mu}\right)=0, \\
\partial_{0} X^{I}-N^{i} \partial_{i} X^{I}=0, & -\partial_{0} P_{I}+\partial_{i}\left(N^{i} P_{I}\right), \\
\partial_{0} A_{i}-N^{j} F_{j i}-\partial_{i} A_{0}=0, & -\partial_{\tau} \pi^{i}+\partial_{j}\left(N^{j} \pi^{i}\right)-\partial_{j}\left(N^{i} \pi^{j}\right)=0, \\
\partial_{i} \pi^{i}=0, & & \\
P_{\mu} \eta^{\mu \nu} P_{\nu}=0, & P_{\mu} \partial_{i} X^{\mu}+P_{I} \partial_{i} X^{I}+p_{T} \partial_{i} T+F_{i j} \pi^{j}=0 .
\end{array}
$$


We would like to see whether these equations of motion have solutions that can be interpreted as the Carroll string moving in the target space-time. To see this we have to determine Carroll limit of fundamental string action, following [35]. We start with the action

$$
S=-\tilde{\tau}_{F} \int d \tau d \sigma \sqrt{-\operatorname{det} G_{\alpha \beta}}, \quad G_{\alpha \beta}=G_{M N} \partial_{\alpha} \tilde{Z}^{M} \partial_{\beta} \tilde{Z}^{N}
$$

Then we obtain

$$
\tilde{K}_{M}=-\tau_{F} g_{M N} \partial_{\beta} \tilde{Z}^{N} G^{\beta \tau} \sqrt{-\operatorname{det} G_{\alpha \beta}}
$$

so that it is easy to see that we have two primary constraints

$$
\mathcal{K}_{\tau}=\tilde{K}_{M} g^{M N} \tilde{K}_{N}+\tilde{\tau}_{F}^{2} \partial_{\sigma} \tilde{Z}^{M} \partial_{\sigma} \tilde{Z}^{N} g_{M N} \approx 0, \quad \mathcal{K}_{\sigma}=\tilde{K}_{M} \partial_{\sigma} \tilde{Z}^{M} \approx 0
$$

and hence the canonical action has the form

$$
S=\int d \tau d \sigma\left(\tilde{K}_{M} \partial_{\tau} \tilde{Z}^{M}-\tilde{\lambda}_{\tau} \mathcal{K}_{\tau}-\tilde{\lambda}_{\sigma} \mathcal{K}_{\sigma}\right)
$$

where $\tilde{\lambda}_{\tau}, \tilde{\lambda}_{\sigma}$ are Lagrange multipliers corresponding to the primary constraints $\mathcal{K}_{\tau}, \mathcal{K}_{\sigma}$. Now we take stringy Carroll limit when

$$
\begin{aligned}
& \tilde{Z}^{\mu}=\frac{Z^{\mu}}{\omega}, \quad \tilde{K}_{\mu}=\omega K_{\mu}, \quad \mu, \nu=0,1, \\
& \tilde{Z}^{I}=Z^{I}, \quad \tilde{K}_{I}=K_{I}, \quad I=2, \ldots, D-1, \\
& \tilde{\tau}_{F}=\omega \tau, \quad \tilde{\lambda}_{\tau}=\frac{\lambda_{\tau}}{\omega^{2}}, \quad \tilde{\lambda}_{\sigma}=\lambda_{\sigma}
\end{aligned}
$$

so that the action has the form

$$
S=\int d^{2} \sigma\left(K_{\mu} \partial_{\tau} Z^{\mu}+K_{I} \partial_{\tau} Z^{I}-\lambda_{\tau}\left(K_{\mu} \eta^{\mu \nu} K_{\nu}+\tau_{F}^{2} \partial_{\sigma} Z^{I} \partial_{\sigma} Z_{I}\right)-\lambda_{\sigma}\left(K_{\mu} \partial_{\sigma} Z^{\mu}+K_{I} \partial_{\sigma} Z^{I}\right)\right) .
$$

The equations of motion that follow from this action have the form

$$
\begin{aligned}
\partial_{\tau} Z^{\mu}-2 \lambda_{\tau} \eta^{\mu \nu} K_{\nu}-\lambda_{\sigma} \partial_{\sigma} Z^{\mu} & =0, & -\partial_{\tau} K_{\mu}+\partial_{\sigma}\left(\lambda_{\sigma} K_{\mu}\right) & =0, \\
\partial_{\tau} Z^{I}-\lambda_{\sigma} \partial_{\sigma} Z^{I} & =0, & -\partial_{\tau} Z_{I}+2 \tau_{F}^{2} \partial_{\sigma}\left(\lambda_{\tau} \partial_{\sigma} Z_{I}\right)+\partial_{\sigma}\left(\lambda_{\sigma} K_{I}\right) & =0, \\
K_{\mu} \eta^{\mu \nu} K_{\nu}+\tau_{F}^{2} \partial_{\sigma} Z^{I} \partial_{\sigma} Z_{I} & =0, & K_{\mu} \partial_{\sigma} Z^{\mu}+K_{I} \partial_{\sigma} Z^{I} & =0 .
\end{aligned}
$$

Comparing (3.18) with (3.11) we immediately obtain that there is no way how solutions of the equations (3.18) could be related to the solution of the equation (3.11) due to the fact that the later contains term proportional to the string tension while such a term is absent in (3.11). In order to resolve this issue we propose Carroll scaling limit when the gauge field scales as well.

\subsection{Modified stringy Carroll scaling limit}

We define this new Carroll scaling limit as

$$
\begin{aligned}
\tilde{x}^{\mu}=\frac{X^{\mu}}{\omega}, & \tilde{p}_{\mu}=\omega P_{\mu}, & \tilde{x}^{I}=X^{I}, & \tilde{p}_{I}=P_{I}, \\
\tilde{A}_{i}=\frac{A_{i}}{\omega}, & \tilde{\pi}^{i}=\omega \pi^{i}, & \tilde{A}_{0}=\frac{1}{\omega} A_{0}, & \tilde{N}=\frac{1}{\omega^{2}} N, \quad \tilde{N}^{i}=N^{i}, \quad \tilde{\tau}_{p}=\omega \tau_{p}, \\
\tilde{T} & =T, & p_{T}=P_{T} . &
\end{aligned}
$$


Then in the limit $\omega \rightarrow \infty\left(g_{M N}=\eta_{M N}\right)$ the the action (3.6) has the form

$$
\begin{aligned}
S=\int d^{p+1} \xi( & P_{\mu} \partial_{0} X^{\mu}+P_{I} \partial_{0} X^{I}+p_{T} \partial_{0} T+\pi^{i} \partial_{0} A_{i}-\pi^{i} \partial_{i} A_{0} \\
& -N\left(P_{\mu} \eta^{\mu \nu} P_{\nu}+\pi^{i} \mathbf{a}_{i j} \pi^{j}+\tau_{p}^{2} V^{2} \operatorname{det} \mathbf{a}_{i j}\right) \\
& \left.-N^{i}\left(P_{\mu} \partial_{i} X^{\mu}+P_{I} \partial_{i} X^{I}+p_{T} \partial_{i} T+F_{i j} \pi^{j}\right)\right)
\end{aligned}
$$

where

$$
\mathbf{a}_{i j}=\partial_{i} X^{I} \partial_{j} X_{I}+\partial_{i} T \partial_{j} T
$$

We see the crucial difference with respect to the previous form of the action (3.8) which is in the presence of the term $\pi^{i} \mathbf{a}_{i j} \pi^{j}$ in the Hamiltonian constraint that is important for string like interpretation of the solutions of the equations of motion at the tachyon vacuum. Explicitly, from (3.20) we obtain following equations of motion

$$
\begin{array}{rlr}
\partial_{0} X^{\mu}-2 N \eta^{\mu \nu} P_{\nu}-N^{i} \partial_{i} X^{\mu}=0, & -\partial_{0} P_{\mu}+\partial_{i}\left(N^{i} P_{\mu}\right)=0, \\
-\partial_{0} P_{I}+2 \partial_{i}\left[N \pi^{i} \delta_{I J} \partial_{j} X^{J} \pi^{j}\right]+2 \tau_{p}^{2} \partial_{i}\left[N V^{2} \delta_{I J} \partial_{j} X^{J}\left(\mathbf{a}^{-1}\right)^{j i} \operatorname{det} \mathbf{a}\right]+\partial_{i}\left(N^{i} P_{I}\right)=0, \\
\partial_{0} X^{I}-N^{i} \partial_{i} X^{I}=0, \\
\partial_{0} T-N^{i} \partial_{i} T=0, \\
-\partial_{\tau} P_{T}+2 \partial_{i}\left(N \pi^{i} \partial_{j} T \pi^{j}\right)+2 \tau_{p}^{2} \partial_{i}\left[N V^{2} \partial_{j} T\left(\mathbf{a}^{-1}\right)^{j i}\right]-2 N \tau_{p}^{2} V \frac{d V}{d T} \operatorname{det} \mathbf{a}_{i j}+\partial_{i}\left(N^{i} p_{T}\right)=0, \\
\partial_{0} A_{i}-N^{j} F_{j i}-\partial_{i} A_{0}-2 N \mathbf{a}_{i j} \pi^{j}=0, & -\partial_{0} \pi^{i}+\partial_{j}\left(N^{j} \pi^{i}\right)-\partial_{j}\left(N^{i} \pi^{j}\right)=0, & \partial_{i} \pi^{i}=0, \\
P_{\mu} \eta^{\mu \nu} P_{\nu}+\pi^{i} \mathbf{a}_{i j} \pi^{j}+\tau_{p}^{2} V^{2} \operatorname{det} \mathbf{a}_{i j}=0, & P_{\mu} \partial_{i} X^{\mu}+P_{I} \partial_{i} X^{I}+p_{T} \partial_{i} T+F_{i j} \pi^{j}=0 .
\end{array}
$$

At the tachyon vacuum these equations of motion simplify considerably and have the form

$$
\begin{aligned}
& \partial_{0} X^{\mu}-2 N \eta^{\mu \nu} P_{\nu}-N^{i} \partial_{i} X^{\mu}=0, \\
& -\partial_{0} P_{\mu}+\partial_{i}\left(N^{i} P_{\mu}\right)=0, \\
& \partial_{0} X^{I}-N^{i} \partial_{i} X^{I}=0, \quad-\partial_{0} P_{I}+2 \partial_{i}\left[N \pi^{i} \delta_{I J} \partial_{j} X^{J} \pi^{j}\right]+\partial_{i}\left(N^{i} P_{I}\right)=0, \\
& -\partial_{0} \pi^{i}+\partial_{j}\left(N^{j} \pi^{i}\right)-\partial_{j}\left(N^{i} \pi^{j}\right)=0 \\
& \partial_{i} \pi^{i}=0, \\
& P_{\mu} \eta^{\mu \nu} P_{\nu}+\pi^{i} \mathbf{a}_{i j} \pi^{j}=0 \\
& P_{\mu} \partial_{i} X^{\mu}+P_{I} \partial_{i} X^{I}+p_{T} \partial_{i} T+F_{i j} \pi^{j}=0 .
\end{aligned}
$$

In order to find string like solutions we proceed in the similar way as in [42]. We introduce a projector

$$
\triangle_{j}^{i}=\delta_{j}^{i}-\frac{\gamma_{j k} \pi^{k} \pi^{i}}{\pi^{m} \gamma_{m n} \pi^{n}}, \quad \gamma_{i j}=\partial_{i} X^{I} \partial_{j} X_{I}
$$

that obeys the property

$$
\triangle^{i}{ }_{j} \pi^{j}=0, \quad \triangle^{i}{ }_{j} \triangle^{j}{ }_{k}=\triangle^{i}{ }_{k}
$$

In other words $\triangle^{i}{ }_{j}$ is the projector on the space transverse to $\pi^{i}$. Then we split $N^{i}$ as

$$
N^{i}=N^{j} \delta_{j}^{i}=\triangle^{i}{ }_{j} N^{j}+\pi^{i} \frac{\pi^{k} \gamma_{k j}}{\pi^{m} \gamma_{m n} \pi^{n}} N^{j}=N_{\perp}^{i}+\pi^{i} N_{I I},
$$


where by definition $N_{\perp}^{i} \gamma_{i j} \pi^{j}=0$. We again introduce dimensionless $\tilde{\pi}^{i}$ defined as $\pi^{i}=$ $\tau_{p} \tilde{\pi}^{i}$ and search for the solution when $\pi^{i}$ is constant. Then the last equation in (3.23) is automatically obeyed while the previous one implies

$$
\tau_{p} \partial_{\sigma} N_{\perp} \pi^{i}-\tau_{p} \partial_{\sigma} N_{\perp}^{i}=0 .
$$

Since this equation has to hold for any $\pi^{i}$ we have to demand that

$$
\partial_{\sigma} N_{\perp}^{i}=0 .
$$

Then it is easy to see that the equations of motion (3.23) have the form

$$
\begin{aligned}
\partial_{0} X^{\mu}-2 N \eta^{\mu \nu} P_{\nu}-N_{I I} \tau_{p} \partial_{\sigma} X^{\mu} & =0, \\
\partial_{0} P_{\mu}+\tau_{p} \partial_{\sigma}\left(N_{I I} P_{\mu}\right) & =0, \\
\partial_{0} X^{I}-\tau_{p} N_{I I} \partial_{\sigma} X^{I} & =0, \\
-\partial_{0} P_{I}+2 \tau_{p}^{2} \partial_{\sigma}\left[N g_{I J} \partial_{\sigma} X^{J}\right]+\tau_{p} \partial_{\sigma}\left(N_{I I} P_{I}\right) & =0
\end{aligned}
$$

that correspond to the equations of motion of the Carroll string (3.18) when we identify $\lambda_{\sigma}=\tau_{p} N_{I I}$ and $\lambda_{\tau} \tau_{F}^{2}=\tau_{p}^{2} N$ (more precise identification will be given in the next section). In other words tachyon vacuum solutions of the stringy Carroll non-BPS Dp-brane can be interpreted as Carroll fundamental string which is in agreement with the general ideas of the tachyon condensation on unstable Dp-brane. Remarkable property of this solution is that it does not depend on all world-volume coordinates of non-BPS Dp-brane but it only depends on $\sigma$, where $\sigma$ is defined by the orientation of the electric flux on the world-volume of nonBPS Dp-brane at the tachyon vacuum. We mean that this is a natural result if we recognize that it is believed that at the tachyon vacuum the non-BPS Dp-brane disappears. Further, note that the localization of the electric flux on the world-volume of non-BPS Dp-brane does not have physical meaning when the full world-volume diffeomorphism invariance is preserved. Of course, this analysis is based on "stringy" Carroll limit when we perform Carroll scaling limit on two coordinates that coincide with the Carroll scaling limit of canonical string action. More interesting situation occurs when we consider Carroll limit à la $p$-brane when we perform contraction in all $p$-spatial coordinates.

Before we proceed to the $p$-Brane Carroll Limit let us solve equations of motion (3.29) in different gauges. As the first case we consider static gauge defined as

$$
X^{0}=\tau, \quad X^{1}=\sigma .
$$

In this gauge the first equation in (3.29) implies

$$
N=-\frac{1}{2 P_{0}}, \quad \tau_{p} N_{I I}=\frac{P_{1}}{P_{0}}
$$

that is of course valid on condition that $P_{0} \neq 0$. To proceed further note that $P_{1}$ can be expressed using the spatial diffeomorphism constraint as

$$
P_{1}=-P_{I} \partial_{\sigma} X^{I}
$$


while $P_{0}$ can be expressed from the Hamiltonian constraint as

$$
P_{0}^{2}=\left(P_{I} \partial_{\sigma} X^{I}\right)^{2}+\tau_{p}^{2} \partial_{\sigma} X^{I} \partial_{\sigma} X_{I}
$$

Now the condition that $P_{0} \neq 0$ implies that $\partial_{\sigma} X^{I} \neq 0$ so that the remaining equations of motion have the form

$$
\begin{aligned}
\partial_{0} X^{I}+\frac{P_{J} \partial_{\sigma} X^{J}}{P_{0}} \partial_{\sigma} X^{I} & =0, \\
\partial_{0} P_{I}-\tau_{p}^{2} \partial_{\sigma}\left[\frac{1}{P_{0}} \partial_{\sigma} X_{I}\right]-\partial_{\sigma}\left[\frac{P_{J} \partial_{\sigma} X^{J} P_{I}}{P_{0}}\right] & =0 .
\end{aligned}
$$

We observe that when we introduce Hamiltonian density $\mathcal{H}_{\text {red }}$ on the reduced phase space defined as

$$
\mathcal{H}_{\mathrm{red}}=-P_{0}=\sqrt{\left(P_{I} \partial_{\sigma} X^{I}\right)^{2}+\tau_{p}^{2} \partial_{\sigma} X^{I} \partial_{\sigma} X_{I}}, \quad H_{\mathrm{red}}=\int d \sigma \mathcal{H}_{\mathrm{red}}
$$

we can write the equations of motion (3.34) into the form

$$
\partial_{0} X^{I}=\left\{X^{I}, H_{\text {red }}\right\}_{\text {red }}, \quad \partial_{0} P_{I}=\left\{P_{I}, H_{\text {red }}\right\}_{\text {red }},
$$

where we defined reduced Poisson brackets

$$
\{X, Y\}_{\mathrm{red}}=\int d \sigma\left(\frac{\partial X}{\partial X^{I}(\sigma)} \frac{\partial Y}{\partial P_{I}(\sigma)}-\frac{\partial Y}{\partial X^{I}(\sigma)} \frac{\partial X}{\partial P_{I}(\sigma)}\right) .
$$

It is easy to see that the time independent configuration corresponds to the situation when $P_{I}=0$ that solves the equation of motion for $P_{I}$ on condition when

$$
\frac{\partial_{\sigma} X^{I}}{\sqrt{\partial_{\sigma} X^{J} \partial_{\sigma} X_{J}}}=k^{I}, \quad k^{I}=\mathrm{const},
$$

where $k^{I}$ obey the equation $k^{I} k_{I}=1$ so that (3.38) has solution $X^{I}=k^{I} \sigma$. We should stress that imposing the static gauge we implicitly work with the infinite extended string so that this configuration corresponds to the straight infinite Carroll string with orientation in the target space-time determined by parameters $k^{I}$.

As the second case we consider the solution in the conformal gauge defined by the condition

$$
N_{I I}=0, \quad N=\frac{1}{2} .
$$

where the equations (3.29) simplify considerably

$$
\begin{aligned}
& \partial_{0} X^{\mu}-\eta^{\mu \nu} P_{\nu}=0, \quad \partial_{0} P_{\mu}=0, \\
& \partial_{0} X^{I}=0, \quad-\partial_{0} P_{I}+\tau_{p}^{2} \partial_{\sigma}^{2} X_{I}=0 . \\
& \eta^{\mu \nu} P_{\mu} P_{\nu}+\partial_{\sigma} X^{I} \partial_{\sigma} X_{I}=0, \quad P_{\mu} \partial_{\sigma} X^{\mu}+P_{I} \partial_{\sigma} X^{I}=0 .
\end{aligned}
$$

The simplest solution corresponds to $X_{I}=c_{I}$ where $c_{I}$ are constants. Then the second equation on the second line in (3.40) implies that $P_{I}=p_{I}(\sigma)$ while from the Hamiltonian 
constraint we find $P_{0}=P_{1}$ where we have chosen the positive branch without lost of generality. Then the spatial diffeomorphism constraint implies

$$
X^{0}+X^{1}=f(\tau)
$$

while the first equation on the first line in (3.40) gives (for $P_{1}=P_{0}$ )

$$
\partial_{0}\left(X^{0}+X^{1}\right)=0
$$

and hence $X^{0}+X^{1}=0$, where we choosen the integration constant to be equal to zero without lost of generality. Finally since $P_{0}=p_{0}(\sigma)$ we find that

$$
\partial_{0}\left(X^{1}-X^{0}\right)=2 p_{0}(\sigma)
$$

that implies $X^{1}-X^{0}=2 p_{0}(\sigma) \tau$ which implies in the end

$$
X^{1}=p_{0} \tau, \quad X^{0}=-2 p_{0} \tau .
$$

In summary, we can find different solutions of the Carroll string equations of motion whose behavior depend on the gauge we impose and on the profile of the initial data.

\section{$3.2 p$-brane Carroll limit}

We studied $p$-brane Carroll limit in section 2. In this section we generalize this analysis to the case of non-zero gauge fields and propose following $p$-brane Carroll limit

$$
\begin{aligned}
& \tilde{x}^{\mu}=\frac{X^{\mu}}{\omega}, \quad \tilde{p}_{\mu}=\omega P_{\mu}, \quad \mu=0, \ldots, p, \\
& \tilde{x}^{I}=X^{I}, \quad \tilde{p}_{I}=P_{I}, \quad I=p+1, \ldots, D-1, \\
& \tilde{A}_{i}=\frac{A_{i}}{\omega}, \quad \tilde{\pi}^{i}=\omega \pi^{i}, \quad \tilde{A}_{0}=\frac{1}{\omega} A_{0}, \\
& \tilde{N}=\frac{1}{\omega^{2}} N, \quad \tilde{N}^{i}=N^{i}, \quad \tilde{\tau}_{p}=\omega \tau_{p}, \\
& \tilde{T}=T, \quad p_{T}=P_{T} .
\end{aligned}
$$

in the action (3.6). Then in the limit $\omega \rightarrow \infty$ we obtain following p-brane Carroll non-BPS Dp-brane action

$$
\begin{aligned}
S=\int d^{p+1} \xi & \left(P_{\mu} \partial_{0} X^{\mu}+P_{I} \partial_{0} X^{I}+p_{T} \partial_{0} T+\pi^{i} \partial_{0} A_{i}-\pi^{i} \partial_{i} A_{0}\right. \\
& -N\left(P_{\mu} \eta^{\mu \nu} P_{\nu}+\pi^{i} \mathbf{a}_{i j} \pi^{j}+\tau_{p}^{2} V^{2} \operatorname{det} \mathbf{a}_{i j}\right) \\
& \left.-N^{i}\left(P_{\mu} \partial_{i} X^{\mu}+P_{I} \partial_{i} X^{I}+p_{T} \partial_{i} T+F_{i j} \pi^{j}\right)\right)
\end{aligned}
$$

where

$$
\mathbf{a}_{i j}=\partial_{i} X^{I} \partial_{j} X_{I}+\partial_{i} T \partial_{j} T .
$$

It is easy to determine equations of motion from the action (3.46) that have formally the same form as the equations of motion (3.22) where now $\mu=0, \ldots, p, I, J=p+1, \ldots, D-1$. 
We are again interested for the analysis of these equations of motion at the tachyon vacuum where they have the form

$$
\begin{aligned}
& \partial_{0} X^{\mu}-2 N \eta^{\mu \nu} P_{\nu}-N^{i} \partial_{i} X^{\mu}=0, \quad-\partial_{0} P_{\mu}+\partial_{i}\left(N^{i} P_{\mu}\right)=0, \\
& \partial_{0} X^{I}-N^{i} \partial_{i} X^{I}=0, \quad-\partial_{0} P_{I}+2 \partial_{i}\left[N \pi^{i} \delta_{I J} \partial_{j} X^{J} \pi^{j}\right]+\partial_{i}\left(N^{i} P_{I}\right)=0, \\
& \partial_{0} A_{i}-2 N \mathbf{a}_{i j} \pi^{j}-N^{j} F_{j i}=0, \quad-\partial_{0} \pi^{i}+\partial_{j}\left(N^{j} \pi^{i}\right)-\partial_{j}\left(N^{i} \pi^{j}\right)=0, \quad \partial_{i} \pi^{i}=0, \\
& P_{\mu} \eta^{\mu \nu} P_{\nu}+\pi^{i} \mathbf{a}_{i j} \pi^{j}=0, \quad P_{\mu} \partial_{i} X^{\mu}+P_{I} \partial_{i} X^{I}+F_{i j} \pi^{j}=0,
\end{aligned}
$$

where we again stress $\mu, \nu=0, \ldots, p, I, J=p+1, \ldots, D-1$. From the Hamiltonian constraint given in (3.48) we see that in order to have string like solution we have to demand that $P_{m}=0$ for $m=2, \ldots, p$. Further, since $\mathbf{a}_{i j}=\partial_{i} X^{I} \partial_{j} X_{I}$ for $I=p+1, \ldots, D-1$ we see that string solution has to be chosen such that $Z^{m}, m=2, \ldots, p$ do not depend on $\sigma$. Then we can formally proceed as in previous section. Firstly we introduce projector

$$
\triangle_{j}^{i}=\delta_{j}^{i}-\frac{\gamma_{j k} \pi^{k} \pi^{i}}{\pi^{i} \gamma_{i j} \pi^{j}}, \quad \gamma_{i j}=\partial_{i} X^{I} \partial_{j} X_{I}
$$

and split $N^{i}$ as

$$
N^{i}=N_{\perp}^{i}+\pi^{i} N_{I I}, \quad N_{\perp}^{i} \gamma_{i j} \pi^{j}=0 .
$$

If we demand that $\pi^{i}=\tau_{p} \tilde{\pi}^{i}$ where $\tilde{p}^{i}$ is constant vector we again find that

$$
\partial_{\sigma} N_{\perp}^{i}=0 .
$$

We further demand that all world-volume field depend on $\sigma$ where $\sigma$ is defined by the relation $\partial_{\sigma}(\ldots)=\tilde{\pi}^{i} \partial_{i}(\ldots)$. In other words we require that $\partial_{i}(\ldots) \triangle^{i}{ }_{j}=0$. Now we are ready to propose following ansatz

$$
\begin{aligned}
& \left.P_{\alpha}\left(\xi^{0}, \sigma\right)\right|_{\xi^{0}=\tau}=\frac{\tau_{p}}{\tau_{F}} K_{\alpha}(\tau, \sigma),\left.\quad X^{\alpha}\left(\xi^{0}, \xi^{1}\right)\right|_{\xi^{0}=\tau}=Z^{\alpha}(\tau, \sigma), \quad \alpha=0,1, \\
& P_{m}=0, \quad Z^{m}=Z^{i}(\tau), \quad m=2, \ldots, p, \\
& \left.X^{I}\left(\xi^{0}, \sigma\right)\right|_{\xi^{0}=\tau}=Z^{I}(\tau, \sigma),\left.\quad P_{I}\left(\xi^{0}, \sigma\right)\right|_{\xi^{0}=\tau}=\frac{\tau_{p}}{\tau_{F}} K_{I}(\tau, \sigma), \quad I=p+1, \ldots, D-1, \\
& \left.N\left(\xi^{0}, \sigma\right)\right|_{\xi^{0}=\tau}=\frac{\tau_{F}^{2}}{\tau_{p}^{2}} \lambda_{\tau}(\tau, \sigma),\left.\quad N^{I I}\left(\xi^{0}, \sigma\right)\right|_{\xi^{0}=\tau}=\lambda_{\sigma}(\tau, \sigma) .
\end{aligned}
$$

Inserting the ansatz (3.52) into equations of motion (3.48) we obtain

$$
\begin{aligned}
& \partial_{\tau} Z^{\alpha}-2 \lambda_{\tau} \eta^{\alpha \beta} K_{\beta}-\lambda_{\sigma} \partial_{\sigma} X^{\alpha}=0, \quad-\partial_{\tau} K_{\alpha}+\partial_{\sigma}\left(\lambda_{\sigma} K_{\alpha}\right)=0, \quad \alpha, \beta=0,1, \\
& \partial_{\tau} Z^{m}=0, \quad K_{m}=0, \\
& \partial_{\tau} Z^{I}-\lambda_{\sigma} \partial_{\sigma} Z^{I}=0, \quad-\partial_{\tau} K_{I}+2 \tau_{F}^{2} \partial_{\sigma}\left[\lambda_{\tau} \delta_{I J} \partial_{\sigma} Z^{J}\right]+\partial_{\sigma}\left(\lambda_{\sigma} K_{I}\right)=0, \\
& K_{\alpha} \eta^{\alpha \beta} K_{\beta}+K_{I} K^{I}+\tau_{F}^{2}\left(\partial_{\sigma} Z^{I} \partial_{\sigma} Z_{I}\right)=0, \quad K_{\alpha} \partial_{\sigma} Z^{\beta}+K_{I} \partial_{\sigma} Z^{I}=0,
\end{aligned}
$$

where in the last step we performed projection of the diffeomorphism constraint $\mathcal{H}_{i}$ to the direction spanned by $\tilde{\pi}^{i}$ and used an anti symmetry of $F_{i j}$ and also used the fact 
that projection of these constraints to the directions transverse to $\tilde{\pi}^{i}$ vanish due to the presumption that all fields depend on $\sigma$ only. In other words (3.52) solve the equations of motion (3.48) on condition that it solves the Carroll string equations of motion which shows that Carroll non-BPS Dp-brane at its tachyon vacuum state is equivalent to the gas of non-interacting Carroll strings with momenta $K_{m}=0$. Of course, the dynamics of these strings is again trivial since the transverse coordinates do not depend on conjugate momenta and are constant on-shell in the gauge $\lambda_{\sigma}=0$. On the other hand the physical meaning of the solutions with $K_{m} \neq 0$ is unclear and deserves further study.

\section{Acknowledgments}

This work was supported by the Grant Agency of the Czech Republic under the grant P201/12/G028.

Open Access. This article is distributed under the terms of the Creative Commons Attribution License (CC-BY 4.0), which permits any use, distribution and reproduction in any medium, provided the original author(s) and source are credited.

\section{References}

[1] S.A. Hartnoll, A. Lucas and S. Sachdev, Holographic quantum matter, arXiv:1612.07324 [INSPIRE].

[2] P. Hořava, Quantum gravity at a Lifshitz point, Phys. Rev. D 79 (2009) 084008 [arXiv:0901.3775] [INSPIRE].

[3] A. Wang, Hořava gravity at a Lifshitz point: a progress report, Int. J. Mod. Phys. D 26 (2017) 1730014 [arXiv:1701.06087] [InSPIRE].

[4] E. Bergshoeff, J. Gomis, B. Rollier, J. Rosseel and T. ter Veldhuis, Carroll versus Galilei gravity, JHEP 03 (2017) 165 [arXiv:1701.06156] [INSPIRE].

[5] E.A. Bergshoeff and J. Rosseel, Three-dimensional extended Bargmann supergravity, Phys. Rev. Lett. 116 (2016) 251601 [arXiv:1604.08042] [INSPIRE].

[6] J. Hartong, Gauging the Carroll algebra and ultra-relativistic gravity, JHEP 08 (2015) 069 [arXiv: 1505.05011] [INSPIRE].

[7] E. Bergshoeff, J. Rosseel and T. Zojer, Newton-Cartan (super)gravity as a non-relativistic limit, Class. Quant. Grav. 32 (2015) 205003 [arXiv: 1505. 02095] [INSPIRE].

[8] R. Andringa, E. Bergshoeff, S. Panda and M. de Roo, Newtonian gravity and the Bargmann algebra, Class. Quant. Grav. 28 (2011) 105011 [arXiv:1011.1145] [INSPIRE].

[9] E. Bergshoeff, J. Rosseel and T. Zojer, Newton-Cartan supergravity with torsion and Schrödinger supergravity, JHEP 11 (2015) 180 [arXiv: 1509.04527] [INSPIRE].

[10] A. Bagchi, Correspondence between asymptotically flat spacetimes and nonrelativistic conformal field theories, Phys. Rev. Lett. 105 (2010) 171601 [arXiv:1006. 3354] [INSPIRE].

[11] A. Bagchi and R. Fareghbal, BMS/GCA redux: towards flatspace holography from non-relativistic symmetries, JHEP 10 (2012) 092 [arXiv: 1203.5795] [INSPIRE]. 
[12] J. de Boer and S.N. Solodukhin, A holographic reduction of Minkowski space-time, Nucl. Phys. B 665 (2003) 545 [hep-th/0303006] [inSPIRE].

[13] G. Arcioni and C. Dappiaggi, Exploring the holographic principle in asymptotically flat spacetimes via the BMS group, Nucl. Phys. B 674 (2003) 553 [hep-th/0306142] [INSPIRE].

[14] G. Barnich and C. Troessaert, Aspects of the BMS/CFT correspondence, JHEP 05 (2010) 062 [arXiv:1001.1541] [INSPIRE].

[15] A. Bagchi, R. Basu, A. Kakkar and A. Mehra, Flat holography: aspects of the dual field theory, JHEP 12 (2016) 147 [arXiv: 1609.06203] [INSPIRE].

[16] H. Bondi, M.G.J. van der Burg and A.W.K. Metzner, Gravitational waves in general relativity. VII. Waves from axi-symmetric isolated systems, Proc. Roy. Soc. Lond. A 269 (1962) 21 [INSPIRE].

[17] R. Sachs, Asymptotic symmetries in gravitational theory, Phys. Rev. 128 (1962) 2851 [INSPIRE].

[18] C. Duval, G.W. Gibbons and P.A. Horvathy, Conformal Carroll groups, J. Phys. A 47 (2014) 335204 [arXiv:1403.4213] [InSPIRE].

[19] C. Duval, G.W. Gibbons and P.A. Horvathy, Conformal Carroll groups and BMS symmetry, Class. Quant. Grav. 31 (2014) 092001 [arXiv:1402.5894] [INSPIRE].

[20] H. Bacry and J. Levy-Leblond, Possible kinematics, J. Math. Phys. 9 (1968) 1605 [InSPIRE].

[21] C. Duval, G.W. Gibbons, P.A. Horvathy and P.M. Zhang, Carroll versus Newton and Galilei: two dual non-Einsteinian concepts of time, Class. Quant. Grav. 31 (2014) 085016 [arXiv: 1402.0657] [INSPIRE].

[22] J. Gomis and H. Ooguri, Nonrelativistic closed string theory, J. Math. Phys. 42 (2001) 3127 [hep-th/0009181] [INSPIRE].

[23] U.H. Danielsson, A. Guijosa and M. Kruczenski, IIA/B, wound and wrapped, JHEP 10 (2000) 020 [hep-th/0009182] [INSPIRE].

[24] J. Gomis, K. Kamimura and P.K. Townsend, Non-relativistic superbranes, JHEP 11 (2004) 051 [hep-th/0409219] [INSPIRE].

[25] J. Gomis, J. Gomis and K. Kamimura, Non-relativistic superstrings: a new soluble sector of $A d S_{5} \times S^{5}, J H E P 12(2005) 024$ [hep-th/0507036] [INSPIRE].

[26] J. Kluson̆, Non-relativistic non-BPS Dp-brane, Nucl. Phys. B 765 (2007) 185 [hep-th/0610073] [INSPIRE].

[27] J. Gomis and P.K. Townsend, The Galilean superstring, JHEP 02 (2017) 105 [arXiv: 1612.02759] [INSPIRE].

[28] C. Batlle, J. Gomis and D. Not, Extended Galilean symmetries of non-relativistic strings, JHEP 02 (2017) 049 [arXiv: 1611.00026] [INSPIRE].

[29] A. Bagchi, Tensionless strings and Galilean conformal algebra, JHEP 05 (2013) 141 [arXiv: 1303.0291] [INSPIRE].

[30] A. Bagchi, S. Chakrabortty and P. Parekh, Tensionless strings from worldsheet symmetries, JHEP 01 (2016) 158 [arXiv:1507.04361] [INSPIRE].

[31] A. Bagchi, S. Chakrabortty and P. Parekh, Tensionless superstrings: view from the worldsheet, JHEP 10 (2016) 113 [arXiv: 1606. 09628] [INSPIRE]. 
[32] E. Bergshoeff, J. Gomis and G. Longhi, Dynamics of Carroll particles, Class. Quant. Grav. 31 (2014) 205009 [arXiv:1405.2264] [INSPIRE].

[33] E. Bergshoeff, J. Gomis and L. Parra, The symmetries of the Carroll superparticle, J. Phys. A 49 (2016) 185402 [arXiv:1503.06083] [InSPIRE].

[34] E.A. Bergshoeff, J. Hartong and J. Rosseel, Torsional Newton-Cartan geometry and the Schrödinger algebra, Class. Quant. Grav. 32 (2015) 135017 [arXiv: 1409.5555] [INSPIRE].

[35] B. Cardona, J. Gomis and J.M. Pons, Dynamics of Carroll strings, JHEP 07 (2016) 050 [arXiv: 1605.05483] [INSPIRE].

[36] A. Sen, Supersymmetric world-volume action for non-BPS D-branes, JHEP 10 (1999) 008 [hep-th/9909062] [INSPIRE].

[37] E.A. Bergshoeff, M. de Roo, T.C. de Wit, E. Eyras and S. Panda, T-duality and actions for non-BPS D-branes, JHEP 05 (2000) 009 [hep-th/0003221] [INSPIRE].

[38] J. Klusoň, Proposal for non-Bogomol'nyi-Prasad-Sommerfield D-brane action, Phys. Rev. D 62 (2000) 126003 [hep-th/0004106] [InSPIRE].

[39] A. Sen, Tachyon dynamics in open string theory, Int. J. Mod. Phys. A 20 (2005) 5513 [hep-th/0410103] [INSPIRE].

[40] A. Sen, Fundamental strings in open string theory at the tachyonic vacuum, J. Math. Phys. 42 (2001) 2844 [hep-th/0010240] [INSPIRE].

[41] A. Sen, Open and closed strings from unstable D-branes, Phys. Rev. D 68 (2003) 106003 [hep-th/0305011] [INSPIRE].

[42] J. Klusoň, Note about unstable D-branes with dynamical tension, Phys. Rev. D 94 (2016) 046004 [arXiv: 1605. 09510] [INSPIRE].

[43] J. Klusoň, Remark about non-BPS Dp-brane at the tachyon vacuum moving in curved background, Phys. Rev. D 72 (2005) 106005 [hep-th/0504062] [INSPIRE]. 